\title{
Study on Strength Training Method in Tennis Course in Universities
}

\author{
Dan Zhang \\ East China Jiaotong University, Nanchang Jiangxi, 330013, China
}

\author{
Key Words: University, Tennis, Course, Strength Training
}

\begin{abstract}
Presently, the strength, speed and capacity in tennis are receiving more and more attention, which become increasingly demanding to tennis players and enthusiasts. Strength is the most basic element of physical quality. Therefore, many tennis educators, including tennis teachers in universities, become much concerned about the strength training method in tennis course. Actually, the intensity of strength training in tennis course in universities is much insufficient, and even some universities have no strength training in tennis course. Thus, it is extremely urgent to intensify strength training in tennis course in universities. In this article, the significance of strength training to tennis learners, the basic factors influencing the strength of tennis learners, and the main methods to intensify strength training have been set out.
\end{abstract}

\section{Introduction}

These tennis players and enthusiasts in universities who want to become a tennis player of high competence must have excellent physical qualifications and quality. As it is known, the parts and muscles of all people engaged in tennis will be affected by the sports to various extents, and some tennis players even suffer body injury due to over training. Through actual survey, it is found that a player will make at least 300 to 500 all-out sprints to finish an official 4-hour tennis game. This implies that good muscular strength is the essential condition for a tennis player of high competence. In order to enhance the muscular strength, the strength training must be intensified.

\section{Significance of Strength Training to Tennis Learners}

First, strength training can help tennis learners to improve their explosive force. As far as a single hitting action is concerned, the learner should coordinate his body parts in a proper order to perform the hitting action, and the muscles on the lower limbs are the core of strength generation. The core area is at the major link of strength transmission, as a connecting link between the preceding and the following. Hence, intensifying the training of the explosive force is favorable for minimizing the strength consumption and loss in the process of strength transmission, and realizing a higher efficiency of strength transmission. Second, strength training can help tennis learners to improve their organizational and coordination ability. Through continued practice for a period, the core strength can be greatly enhanced, which is helpful for improving the coordination ability of body, so that the body will become more stable in movement, and the pressure on the limbs also will be considerably reduced. The realization of tactical movements of tennis requires the whole body coordination of tennis players. Hence, the improvement of coordination ability is very important to the tennis learners to improve the competitive ability. Third, strength training can greatly reduce the probability of body injury. Besides the improvement of tactical ability, intensifying strength training is also helpful for enhancing the elasticity of muscles and ligaments, and the body balance in practice or movements of the tennis learners, which is of great importance in avoiding injury and diseases of tennis players. 


\section{Analysis of Basic Factors Influencing the Strength of Tennis Learners}

\section{Influence on the Nervous System of Tennis Learners}

The control of nervous system refers to raising more muscle fibers to function in movements by increasing the frequency and intensity of incitogram. A survey shows that those tennis players who don't have enough strength training can raise about $60 \%$ muscle fibers only even though they maximize their muscular exertion, while those who have enough regular strength training can raise more than $90 \%$ muscle fibers easily. Generally, the strength and speed of muscular movement are decided by the actual amount of human body movement and the overall contraction of body movement units, and the reaction between the nerve impulse and muscle contraction decides the specific amount of human body movement and the overall contraction. Practice shows that if tennis players employ $20 \%$ to $80 \%$ of their muscular strength in movement, the development of the muscular strength is mainly dependent on the participation of new body units. Once more than $80 \%$ of muscular strength is to be employed, the development of the muscular strength will be dependent on the specific frequency of discharging motor nerve by the nervous system. Hence, it can be seen that the more kinds of muscular fibers are raised by the player, the stronger the force exerted by him is.

\section{Influence on the Mentality of Tennis Learners}

Usually, any movement will inhibit the nervous system of people to a certain extent. Hence, the muscular strength cannot be exerted to the largest extent. That the muscles in the nervous system are controlled is generally caused by the combined action of various psychological factors. For example, the frustration suffered before, fear, injury experience in movement, harsh growth environment, and intense mood all will affect the performance of tennis players. However, if the tennis learners can concentrate on training, and relieve their unhealthy mental emotions by means of psychological hint, they can do much better.

\section{Influence on the Training of Tennis Learners}

The muscular strength training of tennis learners covers intensity conformity, speedy action, movement range, and other aspects, each of which should be repeatedly practiced with different intervals. Generally, proper trainings can improve the muscular strength of the tennis learners considerably. However, trainings are not efforts once for all for players. If the tennis player stops muscular strength training, his strength will gradually decline and degenerate at a speed about one third of the improving speed. That's to say, the speed of improving strength is fast, while the declining speed is also fast. Therefore, the muscular strength state of tennis learners is directly dependent on strength training.

\section{Main Methods to Intensify Strength Training in Tennis Course in Universities}

The tennis learners should develop a modern training perspective. Traditional tennis training mainly focuses on tactical training, and ignores strength training. Thus, totally changing the traditional training perspective, and fully realizing the urgency of strength training of tennis players and their development potential are also an important task in modern tennis training. Meanwhile, the tennis learners should be subject to more intensifier strength training, and trained to realize the importance of strength training, so as to integrate strength training into the training of other competitive sports.

\section{All-round Strength Training}

This training mode applied in tennis course in universities is a main method of strength training of body parts and joints suitable for the learners in early twenties, of which the aim is to laying a solid foundation for further improving the muscular strength and coordination ability of the tennis learners in universities. This training method can be applied for improving the strength of leg, hamstring, hip, chest, shoulder and wrist of players. In order to mobilize the tennis learners to actively participate in 
movements, it is important to conduct all-round strength training. Specifically, this training method covers the following aspects: training of muscular strength, training of muscle endurance, and training with the aid of medicine ball. The training of muscular strength is to be conducted with heavy barbell at a degressive frequency. The postures of barbell training include half squat, back deep squat and bench press. The training of muscle endurance is to be conducted with light barbell at an increasing frequency. For example, the training of wrist strength is conducted by grasping tennis with the biggest strength for 5 to 6 seconds a time, and repeating it 20 times. Medicine ball is useful to realize the intensive training goal via small resistance. Besides, the tennis learners can choose a medicine ball with proper weight based on their age, strength state and physical growth degree. That any tennis learner finds himself unable to make accurate and correct technical motions with a medicine ball through specified trainings implies that he hasn't chosen a proper medicine ball.

\section{Basic Strength Training}

This training mode applied in tennis course in universities is a method of strength training of muscular strength suitable for the learners in early twenties, of which the aim is to constantly improving the explosive force, the speed of shot and muscular coordination of tennis players, and laying a solid foundation for further training of explosive force in future. Besides, this is a also helpful to improve the physical quality and endurance of the learners, so as to prevent injury in movements. This training method covers the following aspects: overcoming body weight, abdominal muscle strength training, and dorsal muscle strength training.

There are many methods of strength training by making use of body weight, including push-up, push-up with breast clapping or hand clapping, monopod push-up, etc. Besides, some training methods with the help of horizontal bar also are available, including pull-up, body twist to the left or right while hands grasping horizontal bar and body hanging, etc. The learners can do the abovementioned trainings by themselves indoor or outdoor to further improve their strength, especially of their upper limbs.

The abdominal muscle strength training aims to help the learners to improve their serve technology and control ability. There are many methods of abdominal muscle strength training, and the learners are better to choose the easier ones. Through enough abdominal muscle strength training, the learners can improve their waist and abdomen strength, to quicken their speed of shot and enhance their explosive force via body twist.

For purpose of the dorsal muscle strength training, two learners compose a group, one person lies flat on the stomach on the ground with hands behind his head, and the elbows separating on both sides and raise his head as best as he can, and the other person presses the legs of the lying person. The lying person should keep this gesture as far as he can. Then, the two persons exchange roles. The times of repetition of each group can be determined by the group members. Through dorsal muscle strength training, the learners can greatly improve their serve speed and strength, and their immunity to back injury.

\section{Strength Training in Combination with Tennis Technique}

The strength training in combination with tennis technique aims to help the learners to integrate their strength into actual combat, and to quicken the speed of swing of the racket and the explosive force. The skills of shot and the combination of skills and strategies are the focal points in this training mode. This training mode mainly covers the following aspects: bounce training, hopping training, explosive force training, and isokinetic strength training.

The bounce ability of players mainly refers to that the muscle group of players constantly stretch, and then contract rapidly, to do powerful actions, and the bounce training is exactly one of the important means to stimulate the explosive force.

The hopping training is a training method combining jumping in place with standing jump. To do the two actions, players should exert the biggest strength, which should be done coherently. The jump distance should not be less than 30 miters, like lateral pyramidal hopping. 
The players should constantly keep doing explosive force training, focusing on it in the general training phase and the specific training phase, reducing the intensity of explosive force training in pre-competition phase, and further reducing the intensity during competition. The most rational training mode is to do it once every two weeks. In this way, the players can save good strength and explosive force for official competitions. In order to avoid interruption of training while going out for competition, the players can take pull strap, chest expander or other auxiliary equipment to do strength training, so as to keep themselves in a good playing condition.

The isokinetic strength training is to be conducted with the help of sports equipment. Different those trainings with the help of portable equipment like dumbbell, the advantage of isokinetic strength training lies in that the players can be free from injury by equipment. Prior to the isokinetic strength training, the teacher should guide the learners to make warm-up exercises for the sake of safety, to avoid body injury in training. The younger learners should properly reduce the times and duration of training. The main aim of training is to improve the strength of body parts of the players, and help them to avoid injury in training.

\section{Conclusion}

In conclusion, strength training is of great significance in tennis course in universities, is important to the improvement of the skills, physical quality and competence of tennis learners and enthusiasts, and can lay a solid foundation for further improvement in future. In all kinds of physical exercises, the core muscle group is regarded as an integrated exercise chain. Whether the core muscle group of a player is powerful determines and supports the exertion of skills and strategies, strength in movements. Besides, through strength training, the tennis learners and enthusiasts can effectively enhance their immunity to injury in movements.

\section{References}

[1] TIAN Maijiu. Theories of Sport Training. Beijing: Higher Education Press, 2006.

[2] YAN Yaxin, CHEN Xiaomeng. Research and Analysis on Core Strength Training in Tennis. Bulletin of Sport Science \& Technology, 2009 (11).

[3] YE Xin. Experiment Research on Core Strength Training of Young Tennis Players. Journal of Foshan University (Natural Science Edition), 2011 (3).

[4] ZHOU Guiqin, YIN Jun. Influence of Vibration Training on the Ability of Curving and Extending of Shoulder Joint of Tennis Players. Journal of Wuhan Institute of Physical Education, 2012 (12).

[5] LI Hui, GAO Xuxiu, ZHANG Pei. Research on Core Strength Training Methods of Tennis Players in Universities. Theories of Sport Training, 2014 (7).

[6] ZHANG Dejun, DING Wei. Research on Core Strength Training Methods for Tennis. Scientific and Technological Information, 2015 (35). 